\title{
Brief Probe on Application of Compass Navigation Satellite System in the Fields of Sea, Land and Air
}

\author{
Yu Zhao \\ Navigation technology Department, Tianjin Maritime College, Tianjin 300350, China \\ email: flykef@163.com
}

Keywords: Compass Navigation Satellite System; the sea, land, aviation field; application

\begin{abstract}
The "Compass Navigation Satellite System" is a navigation satellite system built by China, and it is capable of serving the most parts of the Asia-pacific region. Compass Navigation Satellite System is widely used in the field of navigation, in addition to the common applications such as the accurate positioning, precise timing and brief communication, it also has innovation on constructing digital navigation marks and improving the vessel's cleaning operation level, and so on. The application of Compass Navigation Satellite System in the fields of sea, land and aviation will be discussed in this paper.
\end{abstract}

\section{Introduction}

Compass Navigation Satellite System is a navigation satellite system built by China, operating independently, and it is compatible and shared with other global navigation satellite system in the world. The system is consisted of space constellation part, ground control part and user termination. Compass Navigation Satellite System will provide the stable, reliable and high quality service to the user all over the world, and it will cooperate with other satellite navigation systems to promote the development of global satellite navigation system as well as human civilization and social progress, eventually it will serve the globe and make humankind benefit from it.

\section{Application of Compass Navigation Satellite System in the Field of Navigation}

As everyone knows, Compass Navigation Satellite System is widely used in the field of navigation, in addition to the common applications such as the accurate positioning, precise timing and brief communication, it also has innovation on constructing digital navigation marks and improving the vessel's cleaning operation level, and so on.

The Construction of Digital Navigation Marks using Compass Navigation Satellite System. The modern digital navigation marks is a new navigation marks technology based on computer technology, satellite navigation system, ECDIS, AIS and other new shipping instruments developed. The digital navigation marks constructed based on Compass Navigation Satellite System can link the multi-module communication function of Compass Navigation Satellite System to navigation sensor by net, to form a digital navigation marks network. The design of the network can fully combine the geographical locations, working environment, operation function and working requirement, the combination of Internet and digital navigation marks, geographic location information and the operation information obtained by the digital navigation marks can be transmitted through the network to all AIS receivers, Compass Navigation Satellite system receiver, navigation marks and maritime buoyage and other equipment at sea, making matters-internet [1].

Geographic coordinate information obtained through Compass Navigation Satellite system, collect the information of navigation marks using a state sensor, put the geographic location information, state parameters into an encoding process, and transmit the code information to the computer that equipped with the core of the control module through the communication module. The control center use high capacity computer and other equipment, combined with the electronic chart display and information system, to cope with and to analyze all kinds of information at real-time. Photoelectric control device is arranged in the navigation marks termination, and connected with the AIS master station through network, photoelectric monitoring equipment 
through the light sensor, electric sensor and the humidity sensor to obtain the parameter related the navigation environment, so as to detect the working condition of navigation marks, acquire, store information, and transmit the information stored to the master AIS station through the communication module of the Compass Navigation Satellite system ,such as the measurement to the special state or beacon alarm, the timely transmission of information to the master control station, real-time alarm or warning purpose. Photoelectric control device is mainly composed of a power supply module. In case of special condition or navigation marks alarm, the sensors will transmit the information to the master station to alarm or pre-alarm. Photoelectric monitoring equipment is consisted of core control module, positioning module, communication module, all kinds of sensors, and finally it becomes an embedded application system (as Fig. 1.). On one hand, the system can carry out the full function of a ship borne AIS, on the other hand, it also has the Short Message function derived from Compass Navigation Satellite system so as to monitor the situation of navigation marks, immediate acquire navigation state information, and make the corresponding instruction. The most important of all, navigation marks and AIS master station transmit data through Compass Navigation Satellite system's Short Message function, it has cut down the communication cost and make it simple to achieve.

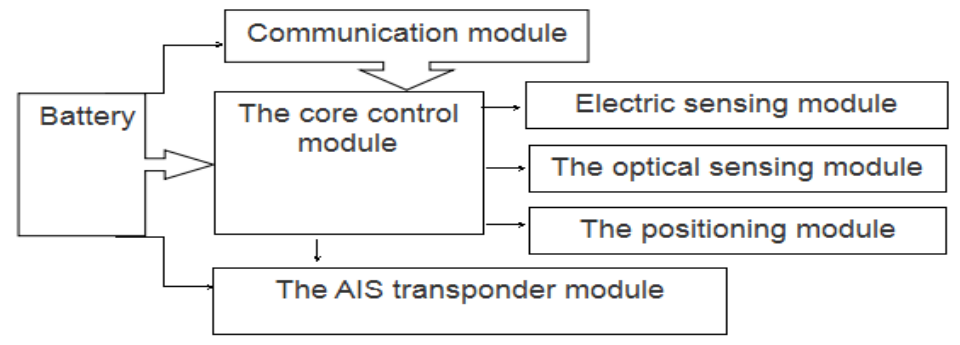

Fig. 1. The structure diagram of digital navigation marks

Improving the Vessel's Cleaning Operation Level Using Compass Navigation Satellite System. By using the bidirectional communication function of Compass Navigation Satellite supervision system can be developed on ship's energy consumption. Vessel's energy consumption level can be obtained by analyzing energy consumption data collection, a ship energy monitoring software can be designed out, and the energy saving scheme of ship can also be calculated so that ship cleaning operation can come true (as follows). Especially with regard to the ships operated in coastal route and inland water routes in china, the Compass Navigation Satellite system's positioning, real-time data acquisition and energy consumption supervision can be combined, using part of the liquefied natural gas instead of high energy consumption and high pollution diesel as the clean energy fuel. Remote wireless monitoring system construction of this dual fuel ship based on Compass Navigation Satellite system, can realize the function of the real-time monitoring of residual natural gas, liquefied natural gas not leak, looking for a nearby coastal gas supply station and so on, can also realize the full aspects, real-time supervision of ship energy consumption, the working condition of ship's main engine and exhaust emission.

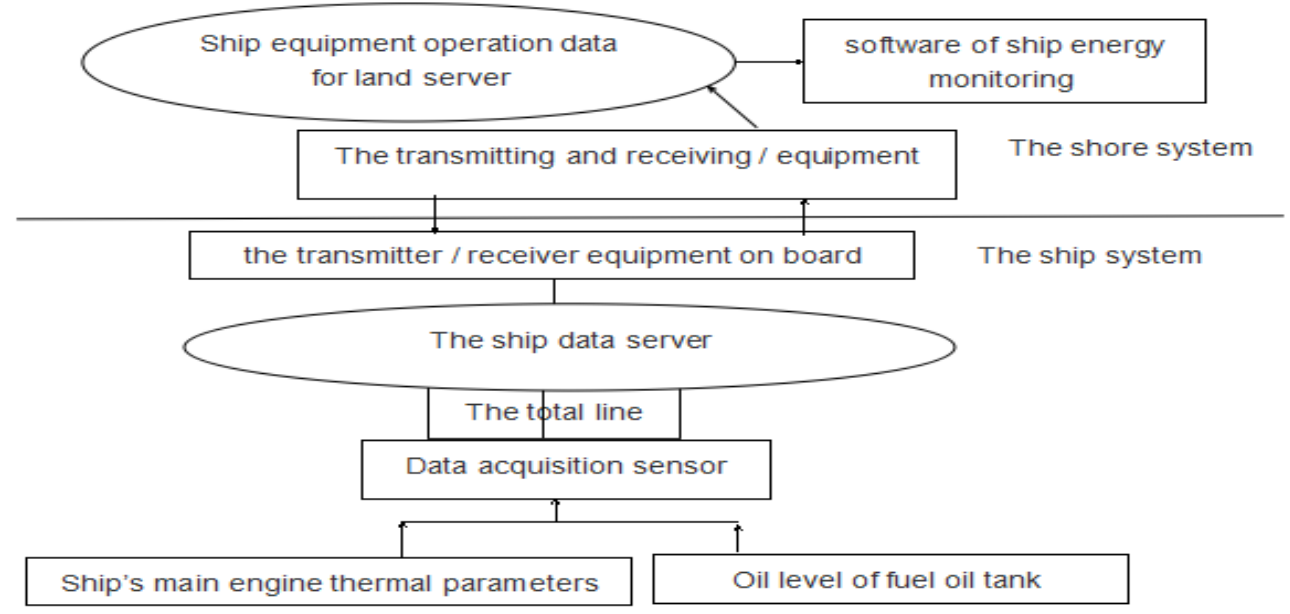

Fig. 2 . Compass Navigation Satellite system assisted ship cleaning operation 


\section{Application of Compass Navigation Satellite System on Land}

Since the Compass Navigation Satellite system has put into use, the satellite navigation application of china has made considerable progress in theoretical research, application technology research and development, manufacturing and application of receiver etc. Compass Navigation Satellite system core chip made in china, module and related key technology have develop so much that they have the same capability as the products of other international countries. Some products have been gradually applied to transportation, forest fire prevention, power dispatching, disaster relief and many other fields, and they have produced a wide range of social and economic benefits. Especially they played an important role in the earthquake in Sichuan Wenchuan, defense applications, farmland monitoring and automatic weather forecasting etc.

Compass Navigation Satellite System Applied in Wenchuan Earthquake. At present, under the promotion of Compass Navigation Satellite system, the electronics, communication, machinery manufacturing, geographic information industry and information service industry in china have developed greatly, showing a significant economic and social benefits. Short message communication function make it possible to implement command immediately. When the Wenchuan earthquake breaking out, mobile phone communication function, all cables, communication equipment all have been destroyed. Under such a situation, the Compass Navigation Satellite system developed a real-time communication lane between the headquarter and the disaster area, played an important role in the medical, rescue and other processes. During the rescue operation, because of its wide coverage of Compass Navigation Satellite system, quick positioning ability and other advantages, it had played an role to assist first rescue personnel and headquarter to rescue the casualties [2].

Defense Applications. The Compass Navigation Satellite system have been widely used and has remarkable effect in various emergency rescue and disaster relief, counter-terrorism, military training aspects since it has been put into use. The huge potential and the special advantage of Compass Navigation Satellite system, not only have completed the military mission, but also are pushing the liberation of information construction [3]. The military functions of Compass Navigation Satellite system is similar to GPS, such as the positioning and navigation for aircraft, missiles, ships and submarines; fast positioning with the launch position for ballistic missile launchers, artillery and excited to launch vehicles and other weapons load to shorten the reaction time; search and rescue operation, mine positioning and mine clearance underwater and so on. This function in the military, means that it can automatically fix the position of any troops, that is to say, once the troops is equipped with the system, in addition to its positioning and navigation function, high command can also master the force position, transmit relevant orders at any time through the system to contribute to the task execution [4].

Farmland Monitoring. There are Many places in China, especially in the northwest, where has been backward farming techniques, lack of water [5]. Agriculture is the main industry in Xinjiang, water resources is the most valuable, there is a great demand for Compass Navigation Satellite system technology collecting data to realize the implementation of precision fertilization, precision irrigation and other agricultural technology [6]. In 2004, Compass Navigation Satellite system was used by the Xinjiang Production Corps for soil moisture data collection, comprehensive utilization of remote data acquisition, positioning system communication technology, remote control technology based on satellite and geographic information technology, realized the real-time monitoring of the geographical position and the temperature and humidity, a comprehensive analysis of drought, and provided an advanced solution for location and land soil moisture, combined with drip irrigation systems, providing an irrigation guidance.

Automatic Forecasting Weather. In order to solve the problem of monitoring and transmission of meteorological data in unmanned area and very cold area, the relevant departments consulted with digital message application experiment, developed the meteorological observation termination equipment based on Compass Navigation Satellite system, and designs the corresponding solution scheme to solve the problem of map display function encountered by the National Meteorological Center and the Bureau of Meteorology visualization of the weather station, meanwhile this device is 
applied in the operation field of weather modification, achieved remarkable results [7]. With the construction and operation of Compass Navigation Satellite system, navigation satellite will be used more widely.

\section{Application of Compass Navigation Satellite System in Aviation Industry}

The Compass Navigation Satellite system of China plays a major role in the sea and land area, it also has the profound influence in the development of the aerospace industry. It is a sign of Chinese aerospace from the construction of infrastructure, the development of space technology stage entered the application stage. At the beginning of Chinese civil aviation , it mainly rely on aviation map for autonomous navigation, due to backward technology, it has brought great troubles to the civil aviation navigation [8]. With the development of Compass Navigation Satellite system and the conditions being ripe, the system are used in many ways in civil aviation navigation service.

Radio Area Navigation (RNAV) of Area Reform based on Satellite Navigation. According to the original definition to area navigation: in the effective scope of navigation facilities on the ground or airborne autonomous navigation system, or in a combination of the above navigation methods which an aircraft can fly through the required air line autonomously and economically [9]. This definition is the design characteristics of ground-based RNAV route currently which navigate relying on the ground navigation system, but this choice is still restricted by the ground navigation station when it is difficult to construct a ground navigation station. The application of BDS, fundamentally shaken off the shackles of ground navigation system when making navigation decisions and makes use of aviation airspace get more rational optimization. with the combination of Compass Navigation Satellite system and the flight management computer, aircraft can fly straightly between two points in the navigation stage in route area, to maximize the choice of flexible, efficient route, to achieve arbitrary route design truly, truly reflects the idea of random navigation [10].

RNP Precision Navigation when Entering and Leaving Airport. RNP, is a new technology to guide the aircraft using its own on board aircraft navigation equipment and global positioning system, and it is new task which developed aviation countries competing to research, and it is also the develop tendency of the aviation in the future. Over the past ten years, RNP has been widely used as a procedure for aircraft to enter and leave the airport. Under the BDS navigation, the plane can fly into the terminal tunnel precisely under the guide of airborne navigation equipment when using the modern flight computer and innovation process. Compared with the traditional navigation technology, the aircraft does not depend on the ground navigation facility that can fly safely along the accurate positioning of the arrival and departure track, and the aircrafts can make precisely landing in poor visibility, accuracy and safety level will be greatly improved. The precisely navigation of Compass Navigation Satellite system is significant for the western plateau airport in our country where terrain is complex, weather is changeable. Precision navigation system can break through the limit of airport related weather standards and minimum descent height for aircrafts, greatly reduce the weather caused flight delays, phenomenon of return, greatly enhanced the airport flight capacity.

Precision Enhancement of Approach and Landing System. At present, the civil airports in our country mainly use the instrument landing system (ILS) as the plane landing means. With the development of the civil aviation, the contradiction between the number of aircraft landing-takeoff and ILS system defect is more and more outstanding [11]. The development of the precisely near landing system which is based on the Compass Navigation Satellite system can solve this contradiction greatly. GLS includes three function modules called precision navigation, flight guidance and landing. GLS can meet the precision approach requirements supported by precise information, and provide the horizontal and vertical deviation guidelines. For ILS, an instrument runway only corresponding to a same class ILS approach procedure, while for GLS, an instrument runway can correspond to a lot of the same level of GLS approach procedures. The required navigation performance ability of the civil aviation airport, GLS can bring obvious approach and 
efficiency and better navigation service when it has a civil airport which is capable of navigating as required. Compare the ground based augmentation system (GBAS) of GLS precision approach and the traditional ILS approach ,GLS has typical advantages, one of which is the approach is more precise, approach and data block can flexibly define the approach path, a GLS system can meet all needs of instrument runway and different types of aircraft landing-takeoff independently in the field, and the approach needs; the second advantage is the stronger environment adaptability, the higher flexibility ,GLS can adapt well when ILS runs difficultly. GLS can support curve approach, the data block can make approach procedure temporarily according to the situation, improve the flexibility of the flight and control.

\section{Conclusions}

The Compass Navigation Satellite system can be used in the sea, land, aviation in different fields, so we can clearly recognize that the system has a wide development prospects. The construction and development of the system will play an essential role for the national security, economic development, science and technology development and social progress.

\section{References}

[1] Zhou Li, Peng Hongchun, Dong Chunlai. [J]. International Space Construction of the "Compass Navigation Satellite System "digital navigation system, 2012 (4).

[2] Ding Chun. Based on the "Compass Navigation Satellite System" ship real-time monitoring system of [J].Navigation technology, 2011 (4).

[3] Satellite navigation and positioning system using [Z]. And Compass Navigation Satellite System’s satellite navigation and positioning Chinese Association, 2012.

[4] Ji Liping. The pseudo satellite technology the "Compass Navigation Satellite System" navigation satellite in regional positioning system $[\mathrm{J}]$ based on. Science of Surveying and mapping, 2002, (4): 53- 57.

[5] Bian Shaofeng, Li Wenkui. Introduction to satellite navigation system[M]. Beijing: Publishing House of electronics industry, 2005.

[6] Ho KC.Chan YT.Geolocation of a known altitude object from TDOA and FDOA measurements [J].IEEE Trans.On Aero-space and Electronic Systems.1997,33 ( 3):770783.

[7] Zhang Mengyang, Summarization for Beidou Satellite Navigation System Application Development[J], Space International, 2009, (11).

[8] Wang Guizhi, fundamental surveying and mapping and "BeiDou" Satellite Navigation Industrialization[A], The Ninth Conference Paper Collection of China Geography Information System[C], 2005.

[9] Caochong,The Industrial Status and Development rospects of GNSS[J], Gnss World of China,2009,(4).

[10] Dou Chang-jiang, The Study on Industrialization of Bei-Dou Navigation Applications[J], Gnss World of China, 2006, (5).

[11] Lu Jing, Zhou yuxia, Zhang Heng, Research on the Beidou Satellite Navigation Standard System[J],Beijing, the First China Satellite Navigation Conference, 2010.05. 